\title{
Identification et analyse qualitative et quantitative d'un combat de lutte olympique
}

\author{
Hasan Melki ${ }^{1}$, Aymen Hawani ${ }^{1}$, Mohamed Sami Bouzid ${ }^{1}$ \\ ${ }^{I}$ (Institut supérieur d'éducation physique et sportive de ksar-said. Tunisie)
}

\begin{abstract}
Résumé: Cette recherche rentre dans le cadre de la logique interne de la didactique de la lutte. Elle permet de préciser le savoir de cette discipline, de cerner précisément ses caractéristiques, ses exigences et les spécificités de sa pratique pour les élèves lutteurs. D'une autre façon, cette étude décrit le contenu de savoir à partir de l'identification et l'analyse qualitative et quantitative d'un combat de lutte. Nous avons fait recours à des mesures directes et nous présentons aux enseignants ainsi qu' aux entraîneurs une méthode d'analyse des séquences temporelles qui pourrait être une nouvelle approche pouvant contribuer à mieux donner des explications à des phénomènes d'apprentissage à travers des paramètres physiologiques et techniques au cours d'un combat de lutte. Vingt lutteurs élèves, de sexe masculin, répartis en 4 groupes, ont participé à l'étude. les résultats contenant les Ratios (effort/repos) debout: $A=1 / 1,63: B=1 / 2,14: C=1 / 1,90: D=1 / 1,10$. Les ratios sol : $A=1 / 1,1: B=1 / 2,62: C=1 / 0,59: D=1 / 1,20$. Tous ces résultats peuvent être considérés comme une référence pour évaluer les capacités du lutteur et spécifier les épreuves de lutte. Aussi elles permettront l'élaboration d'un contenu adapté et structuré pour le présenter aux enseignants et aux entraîneurs afin de le transmettre tout en facilitant l'apprentissage aux lutteurs.
\end{abstract}

Mots clés: sport de combat, savoir de lutte, analyse des séquences temporelles.

\section{Introduction Et Problematique}

Le travail du chercheur n'est pas guidé par le désir de prescrire aux entraineurs ou aux enseignants la façon efficace de penser, car ils n'en n'ont pas besoin, mais pour les informer de ce qui se passe au cours d'une séance d'activité physique qui pourrait être utile pour transmettre un contenu, favoriser l'apprentissage et par la suite améliorer la performance (De Grandmont, 1997). Cette dernière présente le souci de tous les enseignants dans le domaine de l'éducation physique, les entraîneurs et les préparateurs physiques dans le domaine du sport. Toutes ces considérations imposent de chercher de nouvelles approches d'enseignement et d'entraînement pour amener l'élève lutteur, à une situation réussite d'apprentissage et avec l'athlète dans la forme souhaitée le jour de la compétition. Dont le but ultime est d'amener les épaules de l'adversaire au tapis. Récemment, Torres Bonette (2011), à montré que les deux styles de lutte ; lutte libre et gréco-romaine, sont basés sur un système de catégorie de poids qui aspire à protéger la santé des concurrents, limitant autant que possible le risque de blessures et augmentant donc le pourcentage de performance qui dépend de compétences techniques, tactiques et psychologiques.

La pratique de la lutte peut aider à se sentir en sécurité. C'est grâce au sentiment de compétence physique ou corporelle, développé par la pratique d'un sport de combat, que l'élève peut se sentir rassuré. Le combat en général et la lutte en particulier sont vécus comme un moyen de défense. La pratique de la lutte permet de se maîtriser par une meilleure connaissance de ses pouvoirs sur l'autre et un plus grand contrôle des émotions liées à l'affrontement. Bien que, la lutte est considérée comme, un sport complet car la nature de l'opposition entraîne un développement nécessaire de prise et traitement d'informations variées (kinesthésiques et visuelles) en vue de prendre des décisions dans un temps limité et bref. Mais, il n'est pas pratiqué dans les établissements scolaire et ceci peut être expliqué par la difficulté à enseigner ou bien parce qu'elle est intense et nécessite des exigences physiologiques et techniques spécifiques. Cette discipline ne peut pas être recommandée avec sa forme officielle dans le milieu scolaire. Pour cette raison il faut adapter le contenu ou le savoir de cette discipline aux capacités d'apprentissage des élèves tout en gardant les spécificités de cette discipline d'où l'idée de proposer un jeu. En somme il faut scolariser cette activité sportive. Toutefois ce jeu peut être utilisé aussi par les entraineurs de lutte comme un style d'entrainement si on ajoute d'autres paramètres de l'intensité comme la durée de récupération et la durée du travail. C'est à l'entraineur de quantifier et d'entrainer ces lutteurs avec l'intensité souhaitée surtout que ce jeu obéit aux exigences physique de cette discipline et pour changer de la forme classique d'entrainement et de gérer le stress des athlètes surtout quand il s'agit d'une préparation pour un évènement important ou pour un entraînement spécifique aux jeunes lutteurs.

La méthode d'analyse des séquences temporelle pourrait être une nouvelle approche efficace qui contribue à mieux analyser le mouvement et donner des explications à des phénomènes d'apprentissage pour aider l'enseignant ou l'entraineur à adapter le contenu en fonction du temps d'engagement moteur de l'élève lutteur. Pour valider, la nature du contenu de savoir, nous avons fait recours à cette méthode qui mesure le temps 
d'engagement moteur et des spécificités du déroulement de la séance. Cette méthode d'analyse correspond en réalité au temps d'engagement moteur des élèves lutteurs aux cours des combats de simulation. C'est donc en chronométrant les séquences de travail, avec ces différentes natures d'effort; non concurrence, faible te haute intensité. Que l'on pourra analyser et comparer s'ils sont plus actifs lorsqu'ils pratiquent un exercice ou lorsqu'ils pratiquent une activité ludique (ou jeu au sens de " jeux de rivière »). Ce pôle d'analyse, si l'on se réfère aux travaux de Siedentop (1994) peut constituer un bon prédicateur de l'apprentissage car selon lui, plus on pratique de manière effective, plus l'apprentissage est important.

L'objectif de ce travail est de décrire le contenu de savoir à partir de l'identification et l'analyse d'un combat de lutte tant au niveau qualitatif (respect des différentes phases de lutte et des efforts fournis) qu'au niveau quantitatif (durée des séquences de travail, de repos). Ceci dans le but de déclarer ce contenu par les professeurs de lutte en situation d'enseignement, avec une attention plus particulière portant sur la nature du savoir que le professeur souhaite transmettre à ses élèves à partir de la construction des situations d'analyses des séquences temporelles des efforts fournis aux cours des combats de lutte. Nous avons tenté d'analyser les pratiques d'enseignement de la lutte pour savoir ce que les professeurs voulaient transmettre dans les cours de lutte et la manière dont cela était enseigné. Notre propos s'attachera donc à décrire le contenu de savoir qui va déclarer par les professeurs de lutte en situation d'enseignement, avec une attention plus particulière portant sur la nature du savoir que le professeur souhaite transmettre à ses élèves à partir de la construction des situations des analyses des séquences temporelles des efforts fournis aux cours des combats de lutte.

\subsection{Procédures}

\section{Methodologie}

Vingt lutteurs élèves volontaire, de sexe masculin, âgés en moyenne de 18,6 + 2,02 ans, d'un niveau régional et national ont participé à cette étude. Notre expérimentation s'est étalée sur deux semaines, au sein du club des sports individuels à Ettadhamen, de la région de Tunis (Tunisie), au cours desquelles les élèves lutteurs ont réalisé les tests de combats. Les séances d'entraînement qui précède l'évaluation, ont été préparées par l'entraîneur du club, sans intervention du groupe des expérimentateurs. Après avoir expliqué notre protocole, un consentement éclaire a été signé par les sujets et leurs parents selon le comité d'éthique institutionnel approuvé en conformité avec la déclaration de Helsinki (1975).

Les sujets de notre population sont répartis en 4 groupes selon la catégorie de poids:

Groupe « $\mathrm{A} »: \mathrm{n}=04$ combats (8 lutteurs).des lutteurs avec des poids légers (55 kg à $66 \mathrm{~kg}$ )

Groupe «B $» \mathrm{n}=02$ combats (4 lutteurs) des lutteurs avec des poids moyens (66 kg à $74 \mathrm{~kg}$ )

Groupe « $C » n=02$ combats (4 lutteurs) des lutteurs avec des poids lourds (74 kg à $96 \mathrm{~kg}$ )

Groupe « $\mathrm{D} » \mathrm{n}=01$ combat (2 lutteurs) des lutteurs avec des poids lourds plus (96 kg à $120 \mathrm{~kg}$ )

\subsection{Protocole de recherche}

Les combats ont lieu sur un tapis aux normes de la Fédération Internationales de Lutte Associées (FILA) et est arbitré par un arbitre confirmé. Le partenaire du lutteur effectuant le test a pour consigne de s'investir dans un combat de lutte (compétition). Les réponses cardio-ventilatoires étaient mesurées au repos, après un échauffement standardisé de $15 \mathrm{~min}$ et à après 3 minutes de la fin de combat. Toutes les prises de mesures ont été réalisées d'une manière identique avec le même matériel, suivant le même protocole et se sont déroulées dans la même salle de combat.

\subsection{Analyse Vidéo des combats}

Les analyses vidéo ont été réalisées à l'aide d'un logiciel libre et gratuit (Kinovea 0.8.15). Les analyses des combats sont orientées vers la détermination de temps de travail debout et sol et temps de pause. Le temps de travail est caractérisé par trois natures d'effort :

- Effort à haute intensité : caractérisé par les attaques, contre attaques et défenses.

- Effort à faible intensité : caractérisé par la préparation de la prise d'attaque ou de contre.

- Effort caractérisé par le non concurrence : les deux lutteurs dirigent le temps en contact.

- Le temps de repos caractérisé par le non contact.

\subsection{Analyse statistique}

Toutes les analyses statistiques ont été réalisées à l'aide de logiciels statistique « Statistical Package for social Sciences » (SPSS version 16.0) pour comparer les réponses cardio-ventilatoires suscitées lors du combat en fonction des différentes situations. L'ensemble des variables est exprimé par leurs moyennes et leurs écartstypes. On a utilisé l'épreuve «t-test», car dans notre recherche, les participants ont été l'objet de deux mesures et que notre variable dépendante est quantitative. La différence a été considérée significative lorsque p<0.05. 


\subsection{Résultats Anthropométriques:}

\section{Resultats}

La moyenne de la masse corporelle de la population totale est égale à $71 \mathrm{Kg} \pm 18.17 \mathrm{~kg}$. La valeur élevée de l'écart type est due au fait qu'il s'agit d'une groupe des lutteurs masculins appartenant à différentes catégories de poids allant de $55 \mathrm{~kg}$ à $120 \mathrm{~kg}$. La taille de notre population varie de $163 \mathrm{~cm}$ à $183 \mathrm{~cm}$ avec une moyenne de $175.2 \pm 5.75 \mathrm{~cm}$. La moyenne de pourcentage de masse grasse est à l'ordre de $14.42 \pm 6.88 \%$.

\subsection{La lactatémie:}

Dans cette étude nous avons enregistré une évolution de lactate d'une valeur minimale de $2.05 \pm 0.4$ $\mathrm{mmol} / \mathrm{L}$ vers une valeur maximale de $2.85 \pm 0.77$ chez les sujets appartenant aux quatre groupes d'études. 3 minutes après la fin des combats nous avons enregistré une augmentation de lactate chez l'ensemble de nos sujets avec une faible évolution chez les sujets des groupes A et B la concentration passe de $12.50 \pm 2.89$ $\mathrm{mmol} / \mathrm{L}$ vers $12.55 \pm 2.89 \mathrm{mmol} / \mathrm{L}$. chez les deux autres groupes $C$ et $\mathrm{D}$ le taux de lactate évolue vers des valeurs de $13.85 \pm 0.63 \mathrm{mmol} / \mathrm{L}$ comme valeur minimale, et de $15.27 \pm 0.34 \mathrm{mmol} / \mathrm{L}$ comme valeur maximale.

\subsection{Fréquences Cardiaques.}

Nous avons trouvées que la moyenne générale de FC minimal pour le total de la population est de $93.97 \pm 6.8$. Les moyennes de FC minimal de quatre groupes d'études $(\mathrm{A} / \mathrm{B} / \mathrm{C} / \mathrm{D})$ sont d'ordres croissant $(75.38$ $\pm 7.33 / 82,25 \pm 8,65 / 103.25 \pm 9.84 / 115 \pm 1.41)$. La moyenne du groupe « $\mathrm{A}$ » représente la valeur la plus basse et la moyenne du groupe « $\mathrm{D} »$ représente la valeur la plus haute par rapport aux autres groupes.

Pour les FC moyenne, la moyenne générale de toute la population est de $154 \pm 10.2$. Les résultats enregistrés de quatre groupes d'études $(\mathrm{A} / \mathrm{B} / \mathrm{C} / \mathrm{D})$ sont d'ordre suivant $(160.75 \pm 8.66 / 127,25 \pm 20,23 / 161.25$ $\pm 5.56 / 169 \pm 6.36$ ). Les moyennes de FC minimal des groupes « $\mathrm{A}$ et $\mathrm{C}$ » se trouvent similaires et il n' ya pas de différence remarquable. La moyenne de FC minimal du groupe « B » représente la valeur la plus basse par rapport aux autres groupes. Pour le groupe « $\mathrm{D} »$ la moyenne de FC minimal représente la valeur la plus haute enregistrée aux cours des combats de simulation.

La moyenne de FC maximal, de toute la population, enregistré aux cours des combats de simulation est de $191.46 \pm 18.13$. Les moyennes de FC maximal de quatre groupes d'études $(\mathrm{A} / \mathrm{B} / \mathrm{C} / \mathrm{D})$ sont d'ordre suivant ; $(190.12 \pm 5.14 / 171 \pm 37,05 / 196.75 \pm 16.19 / 208 \pm 14.14)$. Les résultats des moyennes de FC maximal, enregistrés montrent que les groupes «A, $\mathrm{C}$ et $\mathrm{D}$ » ont des moyennes tournent au tour la moyenne général de toute la population. Seul le groupe « B » à une moyenne se trouve loin de la moyenne générale.

\subsection{Efforts générales et Effectives.}

L'histogramme $n^{\circ} 1$ résume les durées générales et effectives des combats de simulation des quatre groupes d'études, les résultats enregistrés ont montré que la moyenne des durées effectives de toutes les combats est de 360 secondes, ( $6 \mathrm{~min}$ en total avec 3 rounds de 2 min intercalés par $30 \mathrm{sec}$ de pause entre chaque périodes, selon le règlement de la (FILA). La moyenne des durées générales de quatre groupes d'études est de $466 \pm$ 62.44 secondes. La différence est significative $(\mathrm{t}=14.93$, $\mathrm{ddl}=3$, sig $=0.001)$. La moyenne des durées générales du premier groupe $\mathrm{A}$, est à l'ordre de $512.25 \pm 55.24 \mathrm{sec}$, avec 152 secondes supplémentaire par rapport au temps réglementaire, cela représente un plus de $42 \%$ sur le temps réglementaire. Pour le groupe « B » la moyenne des durées générales est de $415 \pm 7.07 \mathrm{sec}$, avec 115 secondes comme temps supplémentaire qui représente aussi $32 \%$ de temps de plus par rapport au temps réglementaire. Pour groupe « $\mathrm{C} »$ la moyenne est de $528 \pm 5.65$ secondes. la durée générale représente $47 \%$ de temps supplémentaire, Alors que le groupe «D », à une durée générale de 410 secondes, avec $12 \%$ supplémentaires de temps enregistrées par rapport au temps réglementaire.

\subsection{Durées des Séquences de travail et de repos Debout et Sol.}

Les résultats de l'inter-durée des séquences de travail et de repos des 4 groupes sont résumés dans l'histogramme ci-dessous. Les résultats du groupes «A $\mathrm{A}$ ont montré que la somme des durées des séquences debout représentent 241 secondes, La durée des séquences caractérisés par la non concurrence ont une moyenne de 29.33 secondes. Alors que la moyenne des efforts à haute intensité est de 84.5 secondes. Les efforts de faible intensité ont une durée moyenne de 120.6 secondes. Les séquences au sol se défèrent au niveau des moyennes des durées. Les séquences de non concurrence ont une durée moyenne de 6 secondes les efforts de haute intensité et 54.33 secondes comme moyenne de ces séquences. Alors 48.33 secondes est la moyenne des efforts de faible intensité. Le total des durées des moyennes est de 107 secondes. Pour le total des durées des pauses, est de 136 secondes, pour les 4 combats simulées.

\subsection{Nombres des Séquences de travail Debout et Sol.}


L'analyse de l'histogramme $n^{\circ} 7$, refléte le nombre des séquences de travail (debout et sol), selon les groupes d'études. Pour le groupe "A", le nombre total des séquences est de 48 , avec 33 séquences dans la position debout et 15 séquences dans la position sol. Le total des séquences du groupe "'B" est de 41, avec 31 séquences dans la position debout et 10 séquences dans la position sol. . Le total de groupe " $C$ " est de 47, avec 32 séquences dans la position debout et 15 séquences dans la position sol. Alors que pour le groupe " $D$ " le nombre total est de 31 avec 25 séquences dans la position debout et 6 séquences dans la position sol.

\section{Conclusion Et Discussion}

La présente étude rentre dans le cadre de la logique interne de la didactique de la lutte, elle permet de définir le savoir de cette discipline, de cerner précisément ses caractéristiques, ses exigences et les spécificités de sa pratique pour les élèves lutteurs. D'une autre façon, cette étude décrit le contenu de savoir à partir de l'identification et l'analyse d'un combat de lutte tant au niveau qualitatif (respect des différentes phases de lutte et des efforts fournis) qu'au niveau quantitatif (durée des séquences de travail, de repos).

Dans cette étude, nous avons proposé aux enseignants ainsi aux entraîneurs un contenu adapté pour les élèves ainsi aux athlètes suite à des analyses des séquences temporelles, des paramètres physiologiques et techniques au cours d'un combat de lutte. Le savoir transmis par le professeur doit obéir à des variables didactiques (Astolfi, Develay, 1989) et des méthodes d'enseignements favorisant le processus enseignement apprentissage. Cette transmission doit être envisagée, dans une mise en relation entre une instance élève, une instance savoir et une instance enseignante dont l'activité doit elle-même être considérée comme « multifinalisée » (Goigoux, 2007).

Des orientations scientifiques qui produisent des connaissances sur le système didactique à partir d'une posture descriptive et compréhensive, ou encore élaborer des modèles descriptifs et compréhensifs de l'ingénierie du professeur (Brousseau, 1988 ; Sarrazy, 2001 ; Sensevy, 2001). Des orientations praxéologiques qui développent des perspectives d'ingénieries didactiques ou encore des recherches d'aide à la décision (Martinand, 1994).

Cette étude accrédite l'idée que la connaissance et la compréhension des formes d'activité des élèves engagés dans des situations d'apprentissage, et de leurs conditions privilégiées d'émergence, peuvent constituer pour les enseignants des sources d'inspiration fécondes en vue de concevoir des situations d'apprentissage en EP ainsi qu'au cours d'une séance d'entrainement. L'analyse des combats de simulation est un élément essentiel pour distinguer le rapport entre le savoir théorique et le savoir d'actions ou bien les savoirs en usage pour l'enseignement de la pratique de cette discipline.

Toutes ces raisons nous amène à identifier dans un premier temps l'état de forme des lutteurs participant aux combats de simulation et de déterminer le temps d'engagement moteur des lutteurs et des exigences de la lutte par une mesure directe. Ces données peuvent servir comme des indicateurs pour quantifier et adapter le savoir. Dans le contexte de croisement des sciences nous avons mesuré des paramètres physiologiques tels que la fréquence cardiaque comme un indice pour avoir une idée sur l'intensité de l'exercice, l'état de forme ainsi le temps quand l'athlète en plein action durant une séquence de combat.

Nos résultats ont montré que durant des combats de simulation la moyenne de la fréquence cardiaque maximale varie entre $190 \mathrm{bat} / \mathrm{min}$ à $208 \mathrm{bat} / \mathrm{min}$, la moyenne de $\mathrm{FC}$ de quatre groupes est à l'ordre de 191 bat/min. Les lutteurs atteignent en moyen un débit cardiaque minimal qui corresponds à $83-86 \%$ et $96-98 \%$ ces résultats montrent que le combat est une activité fortement concurrentiels, ce qui est confirmé par les résultats (Karmer et al.,2001). Dans ce sens, Horswill et al (1992), a décrit la lutte comme un événement physique intermittent qui produit une grande force et les demandes de puissance de muscle pour les deux membres de corps, inférieur et supérieur, avec une haute demande de métabolisme d'énergie anaérobie. Dans ce même contexte, l'étude de Hrvoje et al (2001) ont montré que la moyenne de battement de cœur avant les combats est de $108 \pm 12$ qui a été légèrement élevée par l'échauffement. Les athlètes ont atteint une fréquence cardiaque de l'ordre de $188 \pm 9,191 \pm 7$ et $192 \pm 9$ suggérant que les combats sont fortement concurrentiels. L'analyse des données des durées effectives des combats du groupe $\langle\mathrm{A} »\langle\mathrm{~B} »\langle\mathrm{C}$ » et $\langle\mathrm{D}$ » est de 360 secondes en moyenne, (6min en total avec 3 rounds de 2 min intercalés par $30 \mathrm{sec}$ de pause entre chaque périodes, selon le règlement de la Fédération International de Lutte Associé (FILA). Concernant les moyennes des durées des groupes A, B, C, D sont respectivement ( $512 \mathrm{sec}, 475 \mathrm{sec}, 528 \mathrm{sec}, 410 \mathrm{sec})$. Ces durées varient entre 410 et 528) ces durées sont supérieures aux durées de temps de travail pendant un combat trouvées récemment de l'étude de Katarzuma (2011) avec une moyenne de $427 \mathrm{sec}$. Nos résultats sont similaires aux résultats de Katarzuma (2011) pour les deux groupes « $\mathrm{C}$ et $\mathrm{D}$ » mais pour les deux autres groupes « $\mathrm{A}$ et $\mathrm{B}$ » nos résultats se trouvent différents.

L'analyse de répartition des points techniques au cours des combats du groupe des légers « $\mathrm{A}$ », donne raison au nombre, plus au moins augmentés, des séquences de travail, on trouve 26 points à la moyenne enregistrée dans les quatre combats, ce qui signifie aussi, que la moyenne des séquences est de 47 séquences, debout et sol, réparties comme suit; séquence à haute intensité est égale à 21 , une séquence à faible intensité est 
égale 21 et une séquence à non concurrence égale à 5 . En ce qui concerne la durée moyenne de séquences caractérisées par la haute intensité est de $79 \pm 11.79$ secondes, représentant $15 \%$ de temps général des combats. Les séquences à faible intensité ont une durée moyenne de $70 \pm 18.04$ secondes, représentant $14 \%$ du temps général des combats. Ceci signifie que les séquences à faible intensité prend la mission des étapes préparatoires des prises d'attaques ou de contre-attaques. En fin la durée moyenne des séquences à non concurrence est de $49.5 \pm 5.46$ secondes, qui représente aussi $10 \%$ du temps générale des combats.

La moyenne des séquences à non concurrence qui est considérée comme la valeur la plus basse des autres, parce que cette phase arrive généralement après les séquences à haute intensité et dans cette phase les lutteurs cherchent à diriger le temps de combat, mais suite aux ordres verbales de l'arbitre qui favorise l'activité et non pas la passivité les lutteurs passent directement à l'action.

L'analyse des résultats du groupe des poids moyens, « $\mathrm{B} »$, donne une raison au nombre plus au moins augmentés de nombres des séquences de travail, on trouve 19 points à la moyenne enregistrée dans les deux combats, ce qui signifie aussi, que la durée moyenne des séquences à haute intensité est de 143 secondes, qui représente $30 \%$ du temps général des combats (475 secondes). Aussi la moyenne des durées des séquences à faible intensité est de 149.5 secondes qui représentent $31 \%$ de temps général des combats. En fin la durée moyenne des séquences à non concurrence est de 88.75 secondes qui représente aussi $18 \%$ du temps générale des combats. L'analyse de nombre des séquences montre que la moyenne des séquences est de 46 séquences, debout et sol, réparties comme suit; séquence à haute intensité est égale à 22 , séquence à faible intensité est égale à 21 , séquence à non concurrence est égale à 3 . L'analyse des sujets du groupe des lourds «C», montre que la moyenne de nombre est de 45.5 séquences, debout et sol, réparties comme suit ; séquence à haute intensité est égale 19 , séquence à faible intensité est égale à 23 , séquence à non concurrence est égale à 3.5 . La moyenne des durées des séquences à haute intensité est de 132.5 secondes, qui représente $25 \%$ du temps générale des combats (528 secondes). Aussi la moyenne des durées des séquences à faible intensité est de 145.56 secondes qui représentent $27.5 \%$ de temps général des combats. En fin la durée moyenne des séquences à non concurrence est de 80.75 secondes qui représente aussi $15 \%$ du temps générale des combats. L'analyse temporelle des sujets du groupe des lourds plus «D», montre que la moyenne de nombre est de 07 séquences, debout et sol, réparties comme suit; séquence à haute intensité est à l'ordre de 3 , séquence à faible intensité est égale 2 , séquence à non concurrence est à l'ordre de 2 . La moyenne des durées des séquences à haute intensité est de 88 secondes, qui représentent $22 \%$ du temps générale des combats ( 410 secondes). Aussi la moyenne des durées des séquences à faible intensité est de 49 secondes qui représentent $23 \%$ de temps général des combats. En fin la durée moyenne des séquences à non concurrence est de 23 secondes qui représentent aussi $6 \%$ du temps générale des combats.

Les analyses de temps de travail et temps de repos, peuvent être considérées comme une référence pertinente pour évaluer les capacités du lutteur et mieux cerner les caractéristiques des épreuves de lutte. Ces derniers aspects sont étroitement liés avec le rendement des lutteurs qui nous donnent ainsi une idée plus claire des facteurs en lien à la performance de l'apprentissage. L'utilisation des connaissances scientifiques par les praticiens est au cœur des réflexions sur la formation et la transmission du contenu.

Finalement, quelque soit la méthode d'entraînement utilisée une analyse d'un combat de lutte est nécessaire pour élaborer un contenu adapté et structuré en tenant compte des savoirs de cette spécialité. De ce fait, nous avons essayé de spécifier le savoir pour le présenter aux enseignants et aux entraîneurs en fonction de la catégorie de poids. Une bonne programmation nécessite une vision détaillée et un savoir spécifique. En fait, ce que nous avons essayé de proposer dans cette étude est une solution pour aider les enseignants à adapter le savoir le lutte aux spécificités et les capacités des lutteurs s'il s'agit d'une séance d'entraînement et de diminuer l'intensité afin de rendre cette activité à une forme modifiée et adaptée aussi plus facile à l'apprentissage dans les établissements.

\section{References}

[1]. Amade-Escot C. (1989) Les stratégies d'enseignement en EPS : contenus proposés, conception de l'apprentissage et perspectives de différenciation, dans Méthodologie et didactique de l'éducation physique et sportive, Clermont-Ferrand, Éditions AFRAPS.

[2]. Amade-Escot C., Barrue J. P., Dugrand M., Terrisse A. (1991). Observatoire des effets de la formation professionnelle par la recherche des enseignants d'EPS : étude de plusieurs groupes de professeurs stagiaires du centre régional de l'académie de Toulouse. Rapport de recherche, Université Toulouse III.

[3]. AMADE-ESCOT C. (2001). - De l'usage des théories de l'enseignement. Questions de l'étude des contrats didactiques en EP. In A. Mercier, G. Lemoyne et A. Rouchier, Le génie didactique. Usages et mésusages des théories de l'enseignement. Bruxelles-Paris : De Boeck.

[4]. Astolfi, J.-P. \&Develay, M. (1989). Didactique des Sciences. Paris, PUF, Que sais-je ?

[5]. Brousseau, G. (1986). Fondements et méthodes en didactique des mathématiques, Recherches en didactique des mathématiques. Grenoble : La Pensée sauvage.

[6]. Baillat G., Demotier A., Espinoza O., Vincent C., Vincent J. (2001) Professeur des écoles au XXIè siècle, réussir son entrée dans le métier, Paris, Éditions Bordas.

[7]. Chateau. (1992), Jouer/Apprendre. Paris : Economica, Anthropos.

[8]. Chevallard, Y. (1991). La transposition didactique. Grenoble, la Pensée Sauvage, nouvelle édition. 
[9]. COLLINET (C.) 2006. «Une analyse sociologique des savoirs scientifiques comme ressources possibles de l'action d'enseignants et d'entraîneurs. ", Revue STAPS, 71, pp. 115-133.

[10]. DOHIN, H. 2001. Mise à l'épreuve d'une option didactique informationnelle de la lutte : le cas d'un cycle d'EPS en sixième, DEA en Sciences de l'éducation, université de Toulouse Le-Mirail.

[11]. DUGAS É. (2003). - Statuts et rôles des pratiques physiques en EPS. In CD-Rom des actes du colloque : 1'ancrage culturel de l'EPS en question. 3es Rencontres de l'EPS. Montpellier.

[12]. Develay, M. (1992). De l'apprentissage à l'enseignement. Paris : ESF.

[13]. DE GRANDMONT, N. (1997). Pédagogie du jeu. Jouer pour apprendre. Paris-Bruxelles : De Boeck \& Larcier.

[14]. FAMOSE J. -P. et al. (1991). - L'apprentissage moteur. Rôle des représentations. Paris : éditions EPS.

[15]. Famose, J-P. (2001). La motivation en éducation physique et en sport. A. Colin, Paris

[16]. Goigoux, R. (2007). Un modèle d'analyse de l'activité des enseignants, Education et didactique, Vol 1/3, 47.

[17]. Horswill CA, Miller JE, Scott JR et al: Anaerobic and aerobic power in arms and legs of elite senior wrestlers. Int J Sports Med, 1992; 13: 558-61.

[18]. Kraemer WJ, Fry AC, Rubin MR et al: Physiological and performance responses to tournament wrestling. Med Sci Sports Exerc, 2001; 33: 1367-78.

[19]. Katarzuma .L: Somatype body composition and proportionality in polish top G.R wrestlers. J human Kinetics.2011.141-154.

[20]. MARTINAND J.-L. (1994). - La didactique des sciences et de la technologie et la formation desenseignants. ASTER, ${ }^{\circ} 19$.

[21]. Maurice sauvageat. (1993). L'activité dans le couple enseignant 1 enseigné ». In Actes de la IXO école d'été de didactique des mathématiques, Association pour la Recherche en Didactique des Mathématiques éditeur, 15-24.

[22]. Millet, G. Le Gallais, D. (2007). Optimisation et limites de la performance sportive. Paris : Masson.

[23]. Mirzaei B, Curby DG, Rahmani-Nia F, Moghadasi M (2009) Physiological profile of elite Iranian junior freestyle wrestlers. J Strength Cond Res 23:2339-2344.

[24]. Piaget, J. (1965). La psychologie de l'intelligence. Paris: Armand Colin ( $8^{\circ}$ édition).

[25]. Revue Française de Pédagogie, ${ }^{\circ}$ 149. 2004.

[26]. SARRAZY B. (2001). - Didactique et enseignement des mathématiques. L'enjeu de leurs rapports pour la formation des professeurs. In A. Mercier, G. Lemoyne et A.Rouchier, Le génie didactique. Usages et mésusages des théories de l'enseignement. Bruxelles-Paris : De Boeck.

[27]. Sauvegrain, J.P. (2001). Analyse didactique de la décision de l'élève. Etude de cas sur l'utilisation du savoir dans un cycle de lutte en Education Physique et Sportive. Thèse (non publiée) mention didactique des disciplines. Université TOULOUSE III - Paul Sabatier

[28]. Sarrazyn, P. Famose, J.P. (2005). Plus c'est difficile et mieux je réussis ! Etude des

[29]. liens entre les buts fixés l'efficacité personnelle et la performance sportive. Bulletin de psychologie, 58 (1), $107-112$.

[30]. SENSEVY G. (2001). - Théories de l'action et action du professeur. In J.-M. Baudoin et J. Friedrich (Eds), Théories de l'action et éducation. Raisons éducatives.Bruxelles : De Boeck.

[31]. SIEDENTOP, D. (1993). Apprendre à enseigner l'éducation physique. (M. Tousignant, P. Boudreau \& A. Fortier, Trad.), Montréal, Gaëtan Morin (oeuvre originale publiée en 1991).

[32]. Terral, P. \& Collinet, C. (2007). L'utilisation des savoirs scientifiques par les enseignants d'EPS : entre description, prescription, justification et méta-cognition. Terrains et travaux, 12. 